\title{
Development of an Adaptive Fuzzy Logic Based Control Law for a Mobile Robot with an Uncalibrated Camera System
}

\author{
T. Das and I.N. Kar \\ Department of Electrical Engineering, Indian Institute of Technology, Delhi, \\ New Delhi- 110016, India \\ ink@ee.iitd.ac.in
}

\begin{abstract}
In this paper, a new adaptive fuzzy controller is proposed for trajectory tracking of wheeled mobile robots by visual servoing. The control algorithm is developed so that it can take care of parametric uncertainty associated with the vision system and the mobile robot dynamics. The system uncertainty associated with nonlinear robot dynamics is estimated by an adaptive fuzzy logic system (FLS) and the uncertain camera parameters are updated online. The controller is designed based on Lyapunov stability theory. Simulation results are presented to illustrate the performance of the proposed controller.
\end{abstract}

\section{Introduction}

Wheeled mobile robots (WMRs) have been used extensively in various industrial and service applications which include security, transportation, inspection and planetary exploration etc. Due to the versatility of applications in various structured and unstructured environments, vision system has been proved to be a very useful sensor for controlling the WMRs. A brief review and some references of vision based tracking and control can be found in [3][2]. It is evident that a model based controller gives rise very good control performance when the sensors are accurately calibrated and the exact model of the system is known. In reality, these two assumptions cease to be valid in many cases. In a vision based robot control system, the parametric uncertainty associated with the calibration of the camera incorporates uncertainty in the WMR position/orientation information. Again, the assumption of having exact models of the robot system means that the robot is not able to adapt to any changes and uncertainties in its models and environment. Hence, the uncertainties discussed above can result in degraded control performance. In this direction, Kelly [7] proposed a set point controller for a planar robot manipulator with uncertain camera calibration parameters. Dixon et.al [3] proposed a trajectory tracking controller for a wheeled mobile robot with uncertain robot dynamics and camera calibration parameters. Recently, Cheah et.al [5]proposed a trajectory tracking controller for a serial link robot manipulator with uncertain robot Jacobian and dynamics. However similar concept has not been applied to the trajectory-tracking problem of a wheeled mobile robot. 
In this paper, a new adaptive control algorithm has been proposed for trajectory tracking by a wheeled mobile robot via visual servoing with uncertain robot dynamics and camera calibration parameters. An adaptive fuzzy logic system (FLS) is capable of uniformly approximating any nonlinear function over compact input space as shown in Wang and Mendel [6]. So, an adaptive FLS has been used to approximate the nonlinear robot dynamics including friction. Thus the controller is robust not only to structured uncertainty such as mass variation but also to unstructured one such as friction. To reduce the error between the value of the real uncertain function and its estimator, we design simple and robust adaptive laws based on Lyapunov stability theory. A velocity transformation matrix, which consists of functions of uncertain camera parameters, relates the velocity of the robot in the task space to the velocity in the camera space. The main new point in this paper is the adaptation to the uncertainty in this velocity transformation matrix in addition to the dynamics uncertainty. Thus the uncertainties in the camera calibration parameters are taken care of. Novel adaptive laws to deal with the uncertainty in the velocity transformation matrix are proposed. The simulation results show the effectiveness of the proposed controller even with nonlinear friction and uncertainty associated with camera calibration.

\section{Basics of Adaptive Fuzzy Logic System}

The basic configuration of a fuzzy system consists of four principal elements: fuzzifier, fuzzy rule base, fuzzy inference engine and defuzzifier. In this paper, multiple-input-single-output (MISO) fuzzy logic systems are considered, because a multiple-output system can always be separated into a collection of single output systems. Following definition is adopted for fuzzy logic system.

Definition 1: The output of a MISO-FLS with singleton fuzzifier, product inference, centroid defuzzifier and Gaussian membership function can be written in the form

$$
f(\boldsymbol{x})=\frac{\sum_{j=1}^{M} \bar{y}^{j}\left(\prod_{i=1}^{n} \mu_{A_{i}^{j}}\left(x_{i}\right)\right)}{\sum_{j=1}^{M}\left(\prod_{i=1}^{n} \mu_{A_{i}^{j}}\left(x_{i}\right)\right)}=\sum_{j=1}^{M} \phi_{j}(x) \bar{y}^{j}=\boldsymbol{r}^{T} \boldsymbol{\Phi}(x)
$$

where $x=\left[x_{1}, \ldots, x_{n}\right]^{T}$ are the input vectors of the fuzzy system, $\bar{y}^{j}$ is the point in the output space $V$ at which $\mu_{B^{j}}\left(y_{l}\right)$ achieves its maximum value and $\mu_{A_{i}^{j}}\left(x_{i}\right)$ is the Gaussian membership function, defined by mean and variance. In the above $\boldsymbol{\Phi}(x)=\left[\phi_{1}(x), \phi_{2}(x), \ldots . ., \phi_{M}(x)\right]^{T} \in \mathfrak{R}^{M}$ is called the fuzzy basis vector [7] and $\boldsymbol{r}=\left[\bar{y}^{1}, \bar{y}^{2}, \ldots, \bar{y}^{M}\right]^{T} \in \mathfrak{R}^{M}$ is called the unknown parameter vector which is to be updated. It has been proved that an adaptive fuzzy logic system is capable of uniformly approximating any real continuous function over a compact input set to 
arbitrary accuracy [6]. In this paper, we use this adaptive fuzzy logic system as an estimator for approximating the functions involving unknown robot parameters.

\section{Kinematics and Dynamics of a Mobile Robot}

The mobile robot is a typical example of a nonholonomic mechanical system. It consists of a vehicle with two driving wheels mounted on the same axis, and a front free wheel. A camera (CCD type) providing an image of the entire robot workspace, is mounted fixed in the ceiling above the robot workspace, such that, (i) its image plane is parallel to the plane of motion of the robot, (ii) the camera can capture images throughout the entire robot workspace. The representation of the mobile robot kinematic model [1][3] in the camera-space takes the following form

$$
\dot{\bar{q}}=S(\bar{q}) \bar{v}
$$

where, $\overline{\boldsymbol{q}}(t)=\left[\begin{array}{lll}\bar{x}_{c}(t) & \bar{y}_{c}(t) \bar{\theta}(t)\end{array}\right]^{T}, \overline{\boldsymbol{v}}(t)=\left[\begin{array}{ll}\bar{v}_{1}(t) & \bar{v}_{2}(t)\end{array}\right]^{T}$ denote the variables.

\subsection{Task Space to Camera-Space Transformation}

We utilize the so-called pin-hole lens model for the robot-camera system to express the robot camera-space position vector in terms of the task-space position vector as shown below [4]

$$
\left[\begin{array}{l}
\bar{x}_{c}(t) \\
\bar{y}_{c}(t)
\end{array}\right]=\boldsymbol{L R}\left(\theta_{0}\right)\left(\left[\begin{array}{l}
x_{c}(t) \\
y_{c}(t)
\end{array}\right]-\left[\begin{array}{l}
O_{o 1} \\
O_{o 2}
\end{array}\right]\right)+\left[\begin{array}{l}
O_{i 1} \\
O_{i 2}
\end{array}\right] \text {, where } \boldsymbol{L}=\left[\begin{array}{cc}
\alpha_{1} & 0 \\
0 & \alpha_{2}
\end{array}\right] \text { with the }
$$

camera constants $\alpha_{1}, \alpha_{2}$. After few algebraic manipulation, we obtain a formulate for a global invertible transformation between the camera-space robot velocities and the task-space robot velocities as follows:

$$
\bar{v}=T_{0} v
$$

where,

$$
\begin{aligned}
& \boldsymbol{T}_{0}=\operatorname{diag}\left(T_{1}, T_{2}\right), T_{1}=\alpha_{1} \cos (\bar{\theta}) \cos \left(\theta+\theta_{0}\right)+\alpha_{2} \sin (\bar{\theta}) \sin \left(\theta+\theta_{0}\right), \\
& T_{2}=\frac{\alpha_{2}}{\alpha_{1}} \cos ^{2}(\bar{\theta}) \cos ^{2}\left(\theta+\theta_{0}\right)+\frac{\alpha_{1}}{\alpha_{2}} \sin ^{2}(\bar{\theta}) \sin ^{2}\left(\theta+\theta_{0}\right)+\frac{1}{2} \sin (2 \bar{\theta}) \sin 2\left(\theta+\theta_{0}\right)
\end{aligned}
$$

The matrix $\boldsymbol{T}_{0}$ is called the velocity transformation matrix. The right hand side of (3) is linear in a set of unknown parameters $\zeta=\left[\zeta_{1}, \ldots, \zeta_{q}\right]^{T}$. Hence, equation (3) can be expressed as $\overline{\boldsymbol{v}}=\boldsymbol{T}_{0}(\overline{\boldsymbol{q}}) \boldsymbol{v}=\boldsymbol{Y}_{v}(\overline{\boldsymbol{q}}, \boldsymbol{v}) \zeta$ where, $\boldsymbol{Y}_{v}(\overline{\boldsymbol{q}}, \boldsymbol{v})$ is called the velocity regressor matrix. Taking into account the equation (3), the dynamic model of the mobile robot is transformed to the following equation: 


$$
\overline{\boldsymbol{M}}(\boldsymbol{q}) \dot{\boldsymbol{v}}+\overline{\boldsymbol{V}}(\boldsymbol{q}, \dot{\boldsymbol{q}}) \boldsymbol{v}+\overline{\boldsymbol{F}}=\overline{\boldsymbol{B}} \tau
$$

where, $\bar{M}=\boldsymbol{S}^{T} \boldsymbol{M S}, \overline{\boldsymbol{V}}=\boldsymbol{S}^{T} \boldsymbol{M} \dot{\boldsymbol{S}}, \overline{\boldsymbol{F}}=\boldsymbol{S}^{T} \boldsymbol{F}$ and $\bar{B}=\boldsymbol{S}^{T} \boldsymbol{B}$.

\section{Control Design}

The primary objective is to force the mobile robot to track a trajectory generated in the camera-space in the presence of parametric uncertainty. The desired trajectory of the robot is generated by a reference model $\dot{\overline{\boldsymbol{q}}}_{r}=\boldsymbol{S}\left(\overline{\boldsymbol{q}}_{r}\right) \overline{\boldsymbol{v}}_{r}$ where, $\overline{\boldsymbol{q}}_{r}(t)$ and $\overline{\boldsymbol{v}}_{r}(t)$ denote the reference trajectory and velocity in the camera-space respectively. Now the problem is to find a smooth velocity control input $\overline{\boldsymbol{v}}_{c}(t)=\boldsymbol{f}_{c}\left(\overline{\boldsymbol{e}}_{p}, \overline{\boldsymbol{v}}_{r}, \boldsymbol{K}\right)$ such that $\lim _{t \rightarrow \infty}\left(\overline{\boldsymbol{q}}_{r}-\overline{\boldsymbol{q}}\right)=0$.

Defining an auxiliary error signal by $\boldsymbol{e}_{p}(t)=\left[\begin{array}{lll}e_{1}(t) & e_{2}(t) & e_{3}(t)\end{array}\right]^{T}$, and auxiliary velocity control $\overline{\boldsymbol{v}}_{c}(t)$ as [1], the auxiliary velocity tracking error in the camera-space is given as $\overline{\boldsymbol{s}}=\overline{\boldsymbol{v}}-\overline{\boldsymbol{v}}_{c}$. In the presence of camera calibration parameters uncertainty, the matrix $\boldsymbol{T}_{0}$ in (4) is uncertain, and under that circumstance, equation (4) can be expressed as

$$
\hat{\bar{v}}=\hat{\boldsymbol{T}}_{0}(\overline{\boldsymbol{q}}, \hat{\zeta}) \boldsymbol{v}=\boldsymbol{Y}_{V}(\overline{\boldsymbol{q}}, \boldsymbol{v}) \hat{\boldsymbol{\zeta}}
$$

where $\hat{\overline{\boldsymbol{v}}}$ denotes an estimated velocity vector in camera-space, $\boldsymbol{T}_{0}(\overline{\boldsymbol{q}}, \hat{\boldsymbol{\zeta}})$ is an approximate velocity transformation matrix and $\hat{\zeta} \in \mathfrak{R}^{q}$ is an estimated kinematic parameters. Defining two variables $\boldsymbol{e}_{c}=\boldsymbol{v}-\boldsymbol{v}_{c}$ and $\boldsymbol{v}_{c}=\hat{\boldsymbol{T}}_{0}^{-1} \overline{\boldsymbol{v}}_{c}$, the error equation is derived as $\overline{\boldsymbol{M}}(\boldsymbol{q}) \dot{\boldsymbol{e}}_{c}+\overline{\boldsymbol{V}}(\boldsymbol{q}, \dot{\boldsymbol{q}}) \boldsymbol{e}_{c}+\boldsymbol{f}(\boldsymbol{x})=\overline{\boldsymbol{B}} \tau$ where, the nonlinear function is $\boldsymbol{f}(\boldsymbol{x})=\overline{\boldsymbol{M}}(\boldsymbol{q}) \dot{\boldsymbol{v}}_{\boldsymbol{c}}+\overline{\boldsymbol{V}}(\boldsymbol{q}, \dot{\boldsymbol{q}}) \boldsymbol{v}_{\boldsymbol{c}}+\overline{\boldsymbol{F}}$ which is approximated by using a FLS as in (1).

\subsection{Proposed Control Law}

we propose an adaptive controller structure, based on the approximate velocity transformation matrix as,

$$
\tau=\overline{\boldsymbol{B}}^{-1}\left(-\hat{\boldsymbol{T}}_{0}^{T}(\overline{\boldsymbol{q}}, \hat{\boldsymbol{\zeta}}) \boldsymbol{K} \hat{\boldsymbol{s}}-\hat{\boldsymbol{T}}_{0}^{T}(\overline{\boldsymbol{q}}, \hat{\zeta}) \boldsymbol{K}_{p} \overline{\boldsymbol{s}}+\hat{\boldsymbol{R}}^{T} \boldsymbol{\Phi}\right)
$$

where $\hat{\bar{s}}=\boldsymbol{Y}_{V}(\overline{\boldsymbol{q}}, \boldsymbol{v}) \hat{\boldsymbol{\zeta}}-\overline{\boldsymbol{v}}_{\boldsymbol{c}}, \overline{\boldsymbol{s}}=\overline{\boldsymbol{v}}-\overline{\boldsymbol{v}}_{c}$ and $\boldsymbol{K}, \boldsymbol{K}_{p}$ are positive definite matrices. The estimated parameter $\hat{\zeta}$ of the approximate velocity transformation matrix $\hat{\boldsymbol{T}}_{0}(\overline{\boldsymbol{q}}, \hat{\boldsymbol{\zeta}})$ and the FLS parameter vector are updated by, 


$$
\begin{aligned}
& \dot{\hat{\boldsymbol{\zeta}}}=\boldsymbol{L}_{V} \boldsymbol{Y}_{V}^{T}(\overline{\boldsymbol{q}}, \boldsymbol{v}) \boldsymbol{K}_{p} \overline{\boldsymbol{s}} \\
& \dot{\hat{\boldsymbol{R}}}=-\Gamma \boldsymbol{\Phi}\left(\boldsymbol{q}, \boldsymbol{v}_{c}, \dot{\boldsymbol{v}}_{c}\right) \boldsymbol{e}_{c}^{T}
\end{aligned}
$$

where $\boldsymbol{L}_{V} \in \mathfrak{R}^{q \times q}$ and $\Gamma$ positive definite design matrices. The closed loop stability of the system is stated in the form of a proposition which can be proved by using a suitable Lyapunov function [8].

Proposition 1: For a nonholonomic mobile robot (4), let the adaptive control law (5) and the parameter update laws (6) be used. Then the system is stable in the sense of Lyapunov. Also, the control law results in the convergence of the position errors $e_{1}$ and $e_{3}$ i.e. $e_{1} \rightarrow 0, e_{3} \rightarrow 0$ and $\overline{\boldsymbol{s}} \rightarrow \boldsymbol{0}$ as $t \rightarrow \infty$.

\section{Simulation Results}

The proposed control law is verified with computer simulation using MATLAB. For simulation purposes, the value of camera calibration parameters, described in equations are taken as $\alpha_{1}=\alpha_{2}=1.0$ pixels $/ m ; \theta_{0}=0.5 \mathrm{rad} ;\left[\begin{array}{ll}O_{o 1} & O_{o 2}\end{array}\right]=\left[\begin{array}{ll}0.2 & 0.1\end{array}\right]^{T} \quad$ and $\left[\begin{array}{ll}O_{i 1} & O_{i 2}\end{array}\right]=\mathbf{0} \in \mathfrak{R}^{2}$. The parameter values given above are selected for simplicity. The parameters of the mobile robot (5) are taken as $m=9 \mathrm{~kg}, I=5.0 \mathrm{kgm}^{2}, r=0.052 \mathrm{~m}, R=0.153 \mathrm{~m}$. The friction term is taken as $\overline{\boldsymbol{F}}=\left[\begin{array}{ll}0.3 \operatorname{sgn}\left(v_{1}\right) & 0.5 \operatorname{sgn}\left(v_{2}\right)\end{array}\right]^{T}$. A circular reference trajectory in the camera-space is taken. The reference trajectory is given by $\bar{x}_{c r}(t)=\cos (t / 20) ; y_{c r}(t)=\sin (t / 20), \bar{\theta}_{r}=\pi / 2+t / 20$; so that reference velocities are given by $\bar{v}_{r 1}=0.05$ pixels $/ \mathrm{sec}, \bar{v}_{r 2}=0.05 \mathrm{rad} / \mathrm{sec}$. The reference trajectory $\mathrm{y}$ in the camera-space starts from $\overline{\boldsymbol{q}}_{r}(0)=\left[\begin{array}{lll}1.0 & 0 & \pi / 2\end{array}\right]^{T}$. The initial posture of the robot in the camera-space is $\overline{\boldsymbol{q}}(0)=\left[\begin{array}{lll}1.1 & 0 & \pi / 2\end{array}\right]^{T}$ and the initial velocities is taken as $v(0)=\left[\begin{array}{ll}0 & 0\end{array}\right]^{T}$. To examine the effects of the uncertainty in camera calibration, the estimated vector $\hat{\zeta}$ is taken as $\hat{\zeta}(0)=\left[\begin{array}{lllll}0.5 & 1.0 & 0.5 & 1.0 & 1.0\end{array}\right]^{T}$. The values of the parameters of the controller are chosen as $k_{1}=20 ; \boldsymbol{K}_{p}=\operatorname{diag}\{35,35\}$; $\boldsymbol{L}_{V}=\operatorname{diag}\{10,10,10,10,10\} ; \Gamma=\operatorname{diag}\{20,20,20\}$. The controller is capable of compensating any sudden change of the robot dynamics because its online learning ability. To demonstrate this, the mass of the mobile robot is kept varying. The mass of the mobile robot is taken as $m=9.0 \mathrm{~kg}$ for $t \leq 70 \mathrm{sec}, m=14.5 \mathrm{~kg}$ for $70<t \leq 130 \mathrm{sec}$ and $m=9.0 \mathrm{~kg}$ for $t>130 \mathrm{sec}$. The simulation results are 


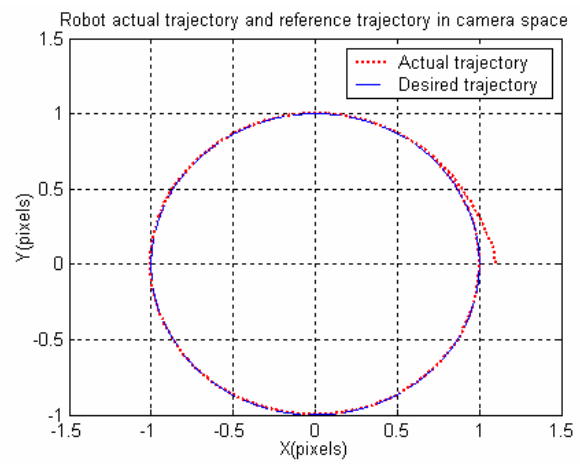

Fig. 1. Performance of the robot in camera-space

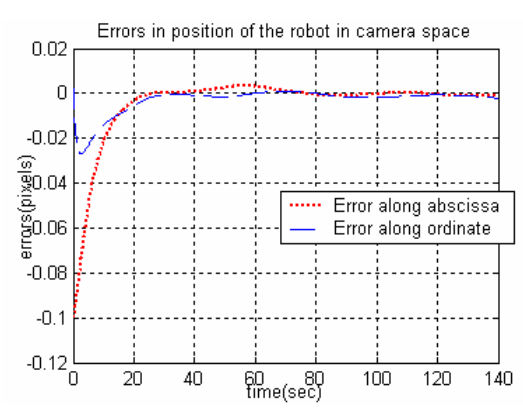

Fig. 2. Tracking errors

shown in figures 1 and 2. Figure 1 show the mobile robot tracks the virtual reference robot moving on the circular path satisfying the nonholonomic constraints, and the tracking errors sharply drop to very small values as shown in Figure 2.

\section{Conclusions}

In this paper, a trajectory tracking control algorithm is proposed for wheeled mobile robots via visual servoing in the presence of parametric uncertainty associated with the camera calibration (e.g. scale factor, focal length, orientation angle) and the nonlinear robot dynamics. An adaptive fuzzy logic system approximates the nonlinear robot dynamics. Novel adaptive laws to deal with the uncertainty in the velocity transformation matrix, which involves functions of uncertain camera parameters, are proposed. Simulation results are presented to illustrate the performance of the proposed adaptive controller.

\section{References}

[1] Y. Kanayama, Y. Kimura, F. Miyazaki, and T. Noquchi, "A stable tracking control method for an autonomous mobile robot," Proc. IEEE Int. Conf. Robotics and automation, vol.1, May 1990, pp. 384-389.

[2] G. Hagar, S. Hutchison, and P. Corke, "A tutorial on visual servo control," IEEE Trans. Robotics and Automation, vol. 12, no.5, 1996, pp. 651-670.

[3] W.E. Dixon, D.M. Dawson, E. Zergeroglu, and Aman Behal, "Adaptive tracking control of a wheeled mobile robot via an uncalibrated camera system," IEEE Trans. Systems, Man, and Cybernetics -Part B: Cybernetics, vol. 31, no.3, June 2001, pp. 341-352.

[4] R.K. Lenz, and R.Y. Tsai, "Techniques for calibration of the scale factor and image center of high accuracy 3-D machine vision metrology," IEEE Trans. Pattern Analysis and Machine Intelligence, vol. 10, no.5, Sep. 1988, pp. 713-720.

[5] C.C. Cheah, C. Liu, and J.J.E. Slotine, "Approximate Jacobian Control for Robot Manipulators," Proc. IEEE Int. Conf. Robotics and Automation, pp. 3075-3080, April 2004. 
[6] Li-Xin Wang, and Jerry M. Mendel, "Fuzzy Basis Functions, Universal Approximation, and Orthogonal Least-Squares Learning," IEEE Trans. Neural Networks, vol. 3, no.5, Sept. 1992, pp. 807-814.

[7] R. Kelly, "Robust asymptotically stable visual servoing of planar robots," IEEE Trans. Robotics and Automation, Vo. 12, pp. 756-766. Oct. 1996.

[8] T. Das, "Intelligent tracking control of robotic systems", MS thesis, Indian Institute of Technology, Delhi, 2005. 